\title{
Tumefactive acute disseminated encephalomyelitis complicating human swine influenza (H1N1)
}

\author{
Amanda CY Chan *, SH Ng
}

\section{A B S T R A C T}

This report illustrates an adult patient presenting with tumefactive acute disseminated encephalomyelitis complicating human swine influenza. Its presentation, diagnosis, investigation findings, course, and response to treatment are discussed herein.
Hong Kong Med J 2014;20:447-50

DOI: 10.12809/hkmj134049

${ }^{1}$ ACY Chan *, FHKCP, FHKAM (Medicine)

${ }^{2} \mathrm{SH} \mathrm{Ng}, \mathrm{FRCP}$ (Lond \& Edin), FHKAM (Medicine)

Department of Medicine and Geriatrics, Tuen Mun Hospital, Tuen Mun, Hong Kong

2 Division of Neurology, Department of Medicine and Therapeutics, The Chinese University of Hong Kong, Prince of Wales Hospital, Shatin, Hong Kong

* Corresponding author: acychan@gmail.com

\section{Introduction}

A new outbreak of influenza caused by a new strain of $\mathrm{H} 1 \mathrm{~N} 1$, also known as 'human swine influenza' was first described in April 2009 in Mexico. This strain was different from the rest, in that it had a propensity to infect very healthy and young subjects, and also caused severe manifestations, such as acute respiratory distress, pneumonia, and even death. Approximately $80 \%$ of affected patients were younger than the age of 25 years. ${ }^{1}$

Since April 2009, there have been few reports of the neurological complications of human swine influenza. ${ }^{2-4}$ We report a case of severe human swine influenza causing acute demyelinating encephalomyelitis of the tumefactive form.

\section{Case report}

A 21-year-old woman with a history of diplegic cerebral palsy and epilepsy was hospitalised for breathlessness, cough, and fever for 3 days in January 2011. The initial chest X-ray was unremarkable, the white cell count (WCC) was elevated $\left(13 \times 10^{9} / \mathrm{L}\right.$; reference range $\left.[R R], 3.4-9.6 \times 10^{9} / \mathrm{L}\right)$ and showed neutrophil predominance $\left(8.9 \times 10^{9} / \mathrm{L}\right.$; RR, 1.27-6.2 x $\left.10^{9} / \mathrm{L}\right)$. An initial nasopharyngeal swab for influenza $A$ and influenza B was negative. She was treated empirically with amoxicillin and clavulanic acid. She developed generalised tonic-clonic convulsion and desaturation 2 days later, for which she was intubated and received intensive care. Computed tomography of the brain showed multiple patchy hypodensities at the grey/white junction and white matter of frontal, parietal and temporal lobes on both sides, suspicious of underlying white matter disease.

Bedside bronchoscopy showed an inflamed mucosa, a small-sized airway with a distorted right bronchus, and purulent sputum. Bronchoalveolar lavage was positive for human swine influenza. The patient was given a course of oseltamivir, and later she received treatment with piperacillin and tazobactam.

One week later, the patient remained comatosed despite discontinuing sedation. Physical examination showed an absent deep pain response, wandering eyes with bilateral tonic pupils, and sluggish response to light. The doll's eye reflex was absent, and the limbs were hypotonic and areflexic.

Autoimmune blood testing revealed nil abnormal. Her erythrocyte sedimentation rate and C-reactive protein level were elevated at $71 \mathrm{~mm} / \mathrm{h}$ (RR, 5-15 mm/h) and $92 \mathrm{mg} / \mathrm{L}(\mathrm{RR}, 0-10 \mathrm{mg} / \mathrm{L})$, respectively. The serum antibody titre for influenza type A showed a significant increase from 10 to 640 over 10 days. Other virus and atypical pneumonia titres were also negative.

Electroencephalogram showed alpha coma pattern, and intermittent generalised slow waves at 1-2 Hz, 50-100 $\mu \mathrm{V}$. Periodic lateralised epileptiform discharges at $1 \mathrm{~Hz}, 40-60 \mu \mathrm{V}$ were evident over the right frontocentral region lasting for 4 to 5 seconds. Overall, the features were supportive of severe encephalopathy and cerebral dysfunction.

Lumbar puncture yielded a high opening pressure, and cerebrospinal fluid (CSF) protein was elevated at $4.26 \mathrm{~g} / \mathrm{L}(\mathrm{RR}, 0.1-0.4 \mathrm{~g} / \mathrm{L})$, glucose 2.2 $\mathrm{mmol} / \mathrm{L}$ (RR, 2.2-3.9 mmol/L), WCC $0.3 \times 10^{6} / \mathrm{L}$, red cell count $0.6 \times 10^{6} / \mathrm{L}$, oligoclonal bands and Gram 


\section{腫脹性急性播散性腦资髓炎併發人類豬型流感} （甲型H1N1流感）感染

\section{陳慈欣、伍仕漢}

本文報告一名腫脹性急性播散性腦脊髓炎併發人類豬型流感的成年患 者，並討論其症狀、診迦、觀察結果、治療和對治療的反應。

stain were negative. Three serial CSF specimens were sent for influenza A viral titres and showed an upward trend. Herpes simplex virus polymerase chain reaction (PCR), tuberculosis PCR, and Cryptococcus were negative.

Magnetic resonance imaging (MRI) of the brain, cervical and thoracic spines showed multiple T1-weighted hypointense, and T2-weighted hyperintense lesions up to $3 \mathrm{~cm}$ in diameter in the cerebral white matter bilaterally, the genu and splenium of the corpus callosum, external and internal capsules, midbrain, dorsal pons, and medulla oblongata. These lesions were contrast non-enhancing. The lesions involving the cerebral white matter and corpus callosum showed ring-like peripheral restricted diffusion.

Long segments of T1-weighted hypointense and $\mathrm{T} 2$-weighted hyperintense lesions were detected along the whole spinal cord, with the cervical cord being the most severely involved. The lesions at the cervical cord were continuous with that at the medulla oblongata (Fig). Overall, the features were in favour of acute disseminated encephalomyelitis (ADEM) with tumefactive demyelination.

This patient was treated with pulsed methylprednisolone, and later given two courses of intravenous immunoglobulin. However, there was no neurological improvement and the patient finally succumbed.

\section{Discussion}

Since the first appearance of human swine influenza
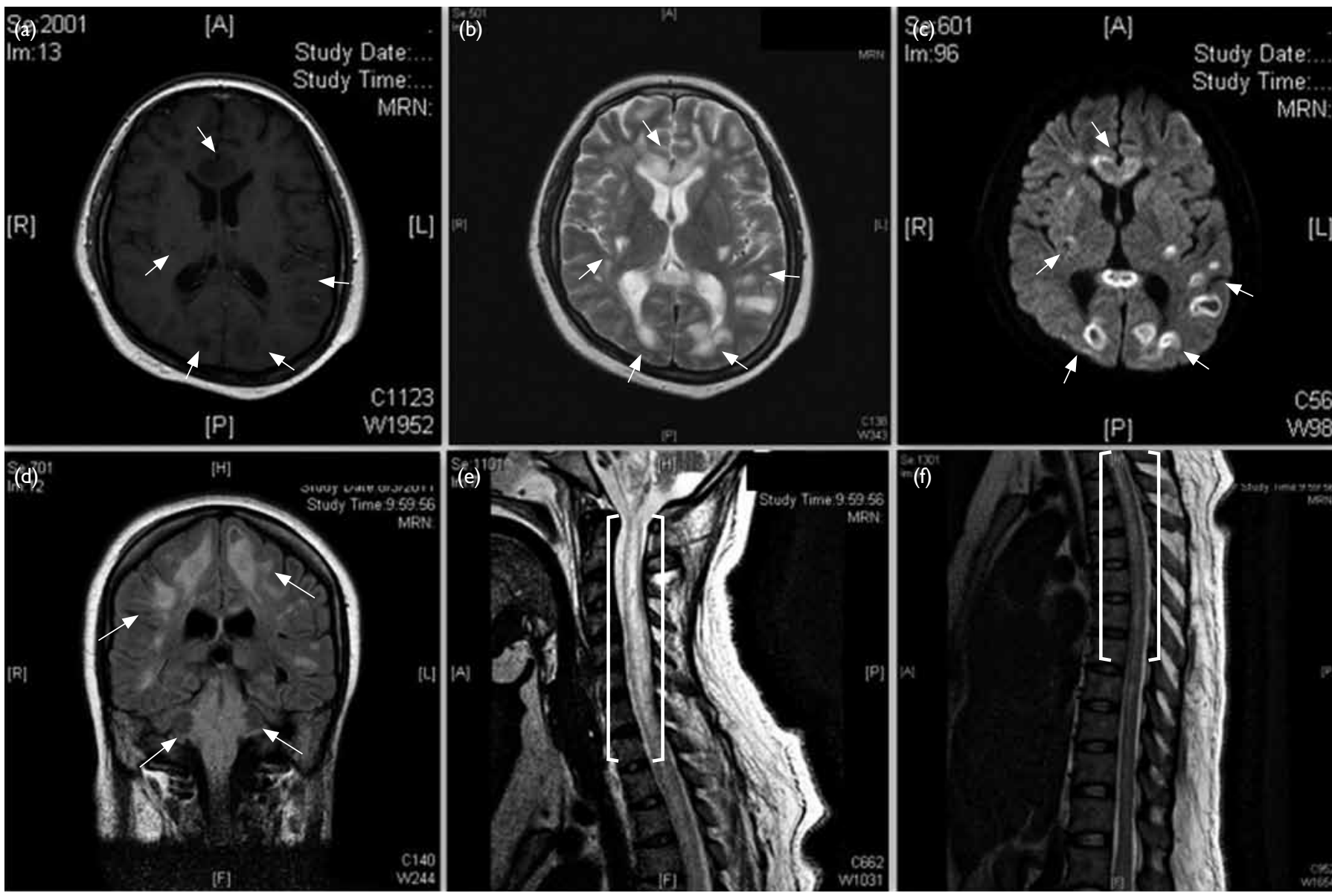

FIG. Magnetic resonance imaging of the (a to d) brain, and (e) cervical and (f) thoracic spines showing multiple ring-like lesions up to $3 \mathrm{~cm}$ in the white matter bilaterally, involving the corpus callosum, external and internal capsules, midbrain, dorsal pons, medulla oblongata (arrows), and along the whole spinal cord (in parentheses). They were (a) TI-weighted hypointense, (b and d) T2-weighted hyperintense, (c) contrast-non-enhancing, with restricted diffusion; collectively in favour of tumefactive acute disseminated encephalomyelitis 
in April 2009 until now, there have been reports of neurological complications that mostly occurred in the paediatric population. A few were also reported in adults, ${ }^{2-4}$ but in them the presentations were not as florid and radiologically severe. We report this case of a 21-year-old, ambulatory and independent woman, with a history of cerebral palsy and epilepsy. She is one of the few adults to have ADEM as a complication of human swine influenza, and the first reported to have the tumefactive form.

Acute disseminated encephalomyelitis is an inflammatory demyelinating disorder of the central nervous system (CNS), which is thought to be due to a T-cell hypersensitivity reaction. ${ }^{5,6}$ It is one of the many syndromes that can develop after vaccination or a microbial infection, and has a 2- to 30-day latency period. ${ }^{3,7}$

The typical MRI appearance is of demyelinating lesions preferentially affecting white matter tracts in a periventricular distribution. Diagnostic difficulty occurs whenever these demyelinating lesions appear to be solitary, large, or tumefactive. Tumefactive lesions are usually defined as solitary lesions, typically greater than $2 \mathrm{~cm}$ in diameter and imaging characteristics resembling a tumour. They tend to be circumscribed and have little in the way of mass effect or vasogenic oedema, typically involving the supratentorium, and are centred within the white matter, although they may extend to involve the cortical grey matter. The exact pathogenesis is unknown. Approximately half of tumefactive demyelinating lesions show pathological contrast enhancement, usually in the form of rings. Commonly they occur in the form of an open ring, with the incomplete portions on the grey matter side of the lesion. The enhancing portion of the ring is believed to represent the leading edge of demyelination and thus favours the white matter side of the lesion. The central non-enhancing core represents a more chronic phase of the inflammatory process. ${ }^{8}$

One must distinguish between infectious and post-infectious encephalitis, as all causes of the former should be excluded before concluding to the latter diagnosis. This involves systemic screening for herpes CNS infections, viruses endemic to specific regions, and other common causes of infective encephalitis. Other mimickers of ADEM include CNS lymphomas, systemic diseases like systemic lupus erythematosus, CNS vasculitis, and vascular, toxic or infectious leukoencephalopathies. ${ }^{5}$ The time course of ADEM, however, is usually hyperacute or acute, whereas the others are usually more chronic. Multiple sclerosis (MS) is also a differential diagnosis, but less likely as in the CSF oligoclonal bands were not present and protein was elevated, and radiologically there were no plaques or lesions disseminated in time. Although in most cases, ADEM is seemingly diagnosed clinically by exclusion, the definitive diagnosis of ADEM is histopathological. Lesions are usually bilateral, although not symmetrical, and mainly they involve the cerebral white matter and brainstem. Occasionally the cerebellum and spinal cord are involved. Small veins and venules in the white matter are surrounded by lymphocytes, macrophages and occasional plasma cells, whereas arteries and arterioles are relatively free of inflammation. Perivascular haemorrhages, axonal fragmentation, inflammatory cells within the leptomeninges, and subpial demyelination in the brainstem and spinal cord may be present. ${ }^{7}$

For our patient, the diagnosis of tumefactive ADEM was mainly made on clinical grounds and typical radiological features. However, postmortem brain biopsy was not performed. We undertook reverse-transcription (RT) PCR analysis for swine flu on CSF samples, but all results came back negative. However, paired CSF and serum samples for influenza A viral titres showed an increasing trend. For patients with suspected neurological complications of swine flu, the sensitivity and specificity of RT PCR and viral titres specifically on CSF samples have not been studied in detail and warrant further investigation. Previous reports of children with influenza A encephalitis in the US showed that CSF PCR were all negative in the three cases. ${ }^{9}$

The treatment of ADEM is borrowed from that of MS. First-line treatment mainly involves corticosteroids, which have been found to shorten the duration of symptoms and halt disease progression. Patients are given 6-methylprednisolone 6 to $8 \mathrm{~g}$ over 6 to 8 days, followed by oral prednisolone at tapering doses, but the prognosis remains variable. Approximately $80 \%$ of patients have a full recovery and ADEM is classically a monophasic disease. However, relapses have been reported in 5\% to $10 \%$ of cases. If relapses occur on more than one occasion, a diagnosis of MS rather than multiphasic disseminated encephalomyelitis is probably more likely. Around $30 \%$ of patients are non-responders to steroids. Half of these non-responders benefit from treatment with intravenous immunoglobulin. Some authors recommend the use of cyclophosphamide in patients at high risk for relapse, either during the first attack or when relapse occurs. However, overall results have been disappointing. ${ }^{10}$

\section{Conclusion}

Herein we report one of the few adult cases of severe tumefactive demyelinating ADEM complicating human swine influenza infection. As different strains of influenza continue to spread throughout the world and in different populations, it is expected that more neurological complications will be reported. As it seems that neurological complications are 
more common in young age-groups with existing neurological diseases, prophylactic vaccination should be considered for such patients. In addition, resorting to early antiviral and immunomodulating therapy should also be emphasised for this patient group.

\section{References}

1. Novel Swine-Origin Influenza A (H1N1) Virus Investigation Team, Dawood FS, Jain S, Finelli L, et al. Emergence of a novel swine-origin influenza A (H1N1) virus in humans. $\mathrm{N}$ Engl J Med 2009;360:2605-15.

2. Kimura E, Okamoto S, Uchida Y, et al. A reversible lesion of the corpus callosum splenium with adult influenzaassociated encephalitis/encephalopathy: a case report. J Med Case Rep 2008;2:220.

3. Athauda D, Andrews TC, Holmes PA, Howard RS Multiphasic acute disseminated encephalomyelitis (ADEM) following influenza type A (swine specific H1N1). J Neurol 2012;259:775-8.
4. Wang J, Duan S, Zhao J, Zhang L. Acute disseminated encephalomyelitis associated with Influenza A H1N1 infection. Neurol Sci 2011;32:907-9.

5. Gupte G, Stonehouse M, Wassmer E, Coad NA, Whitehouse WP. Acute disseminated encephalomyelitis: a review of 18 cases in childhood. J Paediatr Child Health 2003;39:336-42.

6. Ozkale Y, Erol I, Ozkale M, Demir S, Alehan F. Acute disseminated encephalomyelitis associated with influenza A H1N1 infection. Pediatr Neurol 2012;47:62-4.

7. Love S. Demyelinating diseases. J Clin Pathol 2006;59:11519.

8. Given CA 2nd, Stevens BS, Lee C. The MRI appearance of tumefactive demyelinating lesions. AJR Am J Roentgenol 2004;182:195-9.

9. Centers for Disease Control and Prevention (CDC). Neurologic complications associated with novel influenza A (H1N1) virus infection in children-Dallas, Texas, May 2009. MMWR Morb Mortal Wkly Rep 2009;58:773-8.

10. Marchioni E, Tavazzi E, Minoli L, et al. Acute disseminated encephalomyelitis. Neurol Sci 2008;29 Suppl 2:S286-8. 